Portland State University

PDXScholar

3-1-1988

\title{
Limitation of the semirelativistic approach in sum rules and related calculations in atomic physics
}

\author{
M. L. Rustgi \\ P.T. Leung \\ Portland State University
}

S. A. Long

Follow this and additional works at: https://pdxscholar.library.pdx.edu/phy_fac

Part of the Physics Commons

Let us know how access to this document benefits you.

\section{Citation Details}

Rustgi, M. L., Leung, P. T., \& Long, S. T. (1988). Limitation of the semirelativistic approach in sum rules and related calculations in atomic physics. Physical Review A (General Physics), 37(5), 1775-1778.

This Article is brought to you for free and open access. It has been accepted for inclusion in Physics Faculty Publications and Presentations by an authorized administrator of PDXScholar. Please contact us if we can make this document more accessible: pdxscholar@pdx.edu. 


\title{
Limitation of the semirelativistic approach in sum rules and related calculations in atomic physics
}

\author{
M. L. Rustgi and P. T. Leung* \\ Department of Physics, State University of New York, Buffalo, New York 14260
}

S. A. T. Long

National Aeronautics and Space Administration, Langley Research Center, Hampton, Virginia 23665

(Received 14 September 1987)

\begin{abstract}
It is shown that the semirelativistic approach, when applied consistently to atomic calculations involving the Thomas-Reiche-Kuhn sum rule or its applications, may lead to very inaccurate results.
\end{abstract}

In spite of the availability of the fully relativistic (Dirac-type) treatment for atomic calculations, the semirelativistic approach (SA) is still applied many times to problems dealing with inner-shell ionizations ${ }^{1}$ and sum-rule calculations, ${ }^{2-7}$ both because of its simplicity and accuracy. Especially for the latter type of calculations which are encountered in problems on photoabsorption in atomic systems, the SA has been found to be extremely efficient and leads to reasonably accurate results with tremendous simplifications as compared to a fully relativistic state-to-state calculation. ${ }^{8,9}$ The SA or the projection operator approach ${ }^{4}$ is used not just for its simplicity but because of its necessity to get rid of the negative energy states in the sum-rule calculations for a relativistic system, as had been noted first by Levinger, Rustgi, and Okamoto. ${ }^{2}$

In this report, we shall point out that the SA as applied to sum-rule calculations can yield very inaccurate results for the high- $Z$ systems, and that the seemingly good agreement reported earlier is fortuitous due to the application of an approximate eigenstate of the semirelativistic Hamiltonian. The SA can hardly be expected to be very accurate for $Z \simeq 100$ and in general one expects the correction due to the next term, the term of order $(v / c)^{4}$, to be roughly of the order $(\alpha Z)^{4}$, and we shall see that this is indeed the case. We shall limit ourselves mainly to the one-electron atomic systems and shall discuss the implications of our results to the many-electron systems.

We shall start by defining clearly what we mean by the SA. By the SA, we mean that the system is described by the Foldy-Wouthuysen (FW) Hamiltonian to the lowest order in $\beta \equiv v / c$. Thus, for a one-electron system with nuclear charge $Z e$, we have, in standard notation, the eigenvalue equation ${ }^{10,11}$

$$
H_{\mathrm{FW}} \psi_{\mathrm{FW}}=E \psi_{\mathrm{FW}}
$$

where

$$
\begin{aligned}
H_{\mathrm{FW}}= & \frac{\mathrm{p}^{2}}{2 m}+e V-\frac{\mathrm{p}^{4}}{8 m^{3} c^{2}}+\frac{e \hbar}{4 m^{2} c^{2}} \frac{1}{r} \frac{\partial V}{\partial r} \sigma \cdot \mathbf{L} \\
& +\frac{e \hbar^{2}}{8 m^{2} c^{2}} \nabla^{2} V,
\end{aligned}
$$

and

$$
V=-\frac{Z e}{r} .
$$

In Eq. (1), the $\psi_{\mathrm{FW}}$ form a complete set of two-spinor wave functions. The solutions for the eigenenergies of $E$ of Eq. (1) are well known; however, the eigenfunctions $\psi_{\text {FW }}$ are less familiar. ${ }^{10}$ Consequently, in some previous sum-rule calculations ${ }^{2}$ the eigenfunction $\psi_{\mathrm{FW}}$ had been approximated by the hydrogenic Schrödinger-type wave function. Recently, we have discovered that Berestetskii and Landau ${ }^{12}$ (BL) had actually obtained the exact (nonperturbative) form of $\psi_{\mathrm{FW}}$ even before $\mathrm{FW}$ published their work! ${ }^{13}$ The solution by BL can be expressed as ${ }^{11,12}$

$$
\psi_{\mathrm{FW}}(\mathbf{r})=\left(1-\frac{\hbar^{2}}{8 m^{2} c^{2}} \nabla^{2}\right) \phi_{D}(\mathbf{r}),
$$

where $\phi_{D}(\mathbf{r})$ denotes the large components of the wellknown exact Dirac wave function. ${ }^{10}$

In the following, we shall illustrate with some simple examples how these eigenfunctions $\psi_{\mathrm{FW}}$, when consistently applied, may lead to large deviations from the exact relativistic results for hydrogenlike systems with a large nuclear charge $Z$. We shall mainly deal with the ground-state wave function

$$
\phi_{D}^{0}(r)=N r^{\gamma-1} \psi_{S}^{0}(r) \chi_{ \pm},
$$

where $\psi_{S}^{0}(r)$ is the ground-state Schrödinger wave function and is written as $\exp \left(-\mathrm{Zr} / a_{0}\right) ; a_{0}$ denotes the Bohr radius, $\chi_{+}=\left(\begin{array}{l}1 \\ 0\end{array}\right)$ and $\chi_{-}=\left(\begin{array}{l}0 \\ 1\end{array}\right)$ are the up and down spinors, and $\gamma$ is defined as

$$
\gamma=\left[1-(Z \alpha)^{2}\right]^{1 / 2} \text {. }
$$

In (6), $\alpha$ is the fine-structure constant and $N$ in (5) is the normalization constant given by

$$
\begin{aligned}
N= & \frac{1}{2}[\pi \Gamma(2 \gamma+1)]^{-1 / 2}\left(\frac{2 Z}{a_{0}}\right)^{\gamma+1 / 2} \\
& \times\left(1+\frac{1}{4} \frac{\hbar^{2}}{m^{2} c^{2}} \frac{Z^{2}}{a_{0}^{2}} \frac{1}{(2 \gamma-1)}\right)^{-1 / 2} .
\end{aligned}
$$


The $\nabla^{2}$ term in (4) involves the ratio of the kinetic energy to the rest energy of the atomic electron and hence should be negligible for small $Z$. However, this term may contribute significantly to the high- $Z$ systems.

As illustrations, let us calculate the mean-square radius $\left\langle r^{2}\right\rangle$ and form factor $F(q, Z)$ for hydrogenlike systems. The mean-square radius is known to be related to the bremsstrahlung-weighted cross section. ${ }^{14}$ From Eqs. (4) and (5), it is straightforward to show that

$$
\begin{aligned}
\left\langle r^{2}\right\rangle_{\mathrm{SA}} & =\int \psi_{\mathrm{FW}}^{0 *} r^{2} \psi_{\mathrm{FW}}^{0} d \mathbf{r} \\
& =4 \pi N^{2}\left(\frac{a_{0}}{2 Z}\right]^{2 \gamma+3} \Gamma(2 \gamma+3)\left[1-\frac{1}{4} \frac{\hbar^{2}}{m^{2} c^{2}}\left[\frac{Z}{a_{0}}\right]^{2} \frac{1-3 \gamma}{(1+\gamma)(1+2 \gamma)}\right]
\end{aligned}
$$

and

$$
\begin{aligned}
F_{\mathrm{SA}}(q, Z)= & \int \psi_{\mathrm{FW}}^{0 *} e^{i \mathrm{q} \cdot \mathrm{r}} \psi_{\mathrm{FW}}^{0} d \mathbf{r} \\
= & \frac{4 \pi}{q} N^{2}\left[\frac{\Gamma(2 \gamma)}{\left[\left(\frac{2 Z}{a_{0}}\right]^{2}+q^{2}\right]^{\gamma}}\left[1-\frac{\hbar^{2}}{4 m^{2} c^{2}} \frac{Z^{2}}{a_{0}^{2}}\right] \sin \left[2 \gamma \tan ^{-1}\left[\frac{q a_{0}}{2 Z}\right]\right)\right] \\
& -(\gamma-1) \frac{\Gamma(2 \gamma-2)}{\left.\left[\left(\frac{2 Z}{a_{0}}\right]^{2}+q^{2}\right]^{\gamma-1} \sin \left[2(\gamma-1) \tan ^{-1}\left(\frac{q a_{0}}{2 Z}\right)\right]\right)} .
\end{aligned}
$$

On the other hand, on using the exact Dirac wave function, ${ }^{6}$ we get the fully relativistic results

$$
\left\langle r^{2}\right\rangle_{R}=2\left(a_{0} / 2 Z\right)^{2}(2 \gamma+1)(\gamma+1)
$$

and

$$
\begin{aligned}
F_{R}(q, Z)= & \frac{1}{(2 \gamma q)}\left(\frac{2 Z}{a_{0}}\right]^{2 \gamma+1} \frac{1}{\left[\left(\frac{2 Z}{a_{0}}\right)^{2}+q^{2}\right]^{\gamma}} \\
& \times \sin \left[2 \gamma \tan ^{-1}\left(\frac{q a_{0}}{2 Z}\right)\right] .
\end{aligned}
$$

We have carried out numerical calculations for each of the results in Eqs. (8)-(11). A comparison of $\left\langle r^{2}\right\rangle_{S A}$ and $\left\langle r^{2}\right\rangle_{R}$ is shown in Table I. It can be seen from Table I that the SA yields considerably smaller values of the mean-square radius in comparison with the relativistic ones. These results differ by as much as $25 \%$ for $Z=100$.

The results for the form factor, however, do not show any significant differences. This is so because for small values of $q, F(q, Z) \cong 1-q^{2}\left\langle r^{2}\right\rangle / 6$, and though $\left\langle r^{2}\right\rangle_{\mathrm{SA}}$ and $\left\langle r^{2}\right\rangle_{R}$ differ significantly for large $Z$, the deviation of
$F(q, Z)$ from 1 is almost negligible because of the presence of $q^{2}$. For large values of $q$, the exponential factor in the expression for the form factor oscillates so rapidly that the form factor becomes very small and almost identical in both the approximations. This example explains to a certain extent the occasional success of the SA.

As a more interesting example, let us refer to the calculation of the real part of the forward scattering amplitude $f(\omega)$ for a $K$-electron in the high-photon-energy limit. ${ }^{2}$ While numerical results from an exact Dirac-type calculation are available, ${ }^{15}$ the problem has also been treated by the much simpler sum-rule approach. Nonrelativistically one would just expect, in units of $e^{2} / m c^{2}$, employing the Thomas-Reiche-Kuhn (TRK) sum rule, ${ }^{16}$

$$
f(\infty)=1 \text {. }
$$

TABLE I. Comparison of the mean-square radius using semirelativistic and relativistic wave functions.

\begin{tabular}{rcc}
\hline \hline$Z$ & $\left\langle r^{2}\right\rangle_{\mathrm{SA}}$ & $\left\langle r^{2}\right\rangle_{R}$ \\
\hline 10 & $8.363 \times 10^{-19}$ & $8.375 \times 10^{-19}$ \\
40 & $4.873 \times 10^{-20}$ & $4.987 \times 10^{-20}$ \\
60 & $1.949 \times 10^{-20}$ & $2.067 \times 10^{-20}$ \\
80 & $9.147 \times 10^{-21}$ & $1.040 \times 10^{-20}$ \\
100 & $4.093 \times 10^{-21}$ & $5.579 \times 10^{-21}$ \\
\hline
\end{tabular}




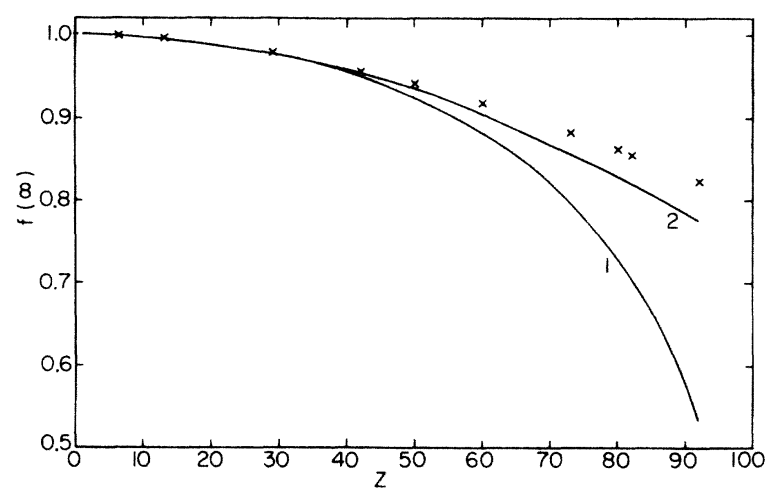

FIG. 1. Comparison of the scattering factor $f(\infty)$ for oneelectron systems employing the semirelativistic approach (curve 1), the Schrödinger-type wave functions (curve 2), and the exact relativistic calculation (crosses).

The correction employing the SA gives ${ }^{2}$

$$
f(\infty)=1-\langle 0|T| 0\rangle / m c^{2}+O\left[\left(\langle 0|T| 0\rangle / m c^{2}\right)^{2}\right],
$$

where $T$ is the kinetic-energy operator and $|0\rangle$ is given by Eqs. (4) and (5). Previously, the ground-state kinetic energy had been evaluated by employing the hydrogenic Schrödinger wave function. Here we wish to show that on using the exact ground-state wave function as given in Eqs. (4) and (5), one obtains significantly different results for the heavy atoms. Thus, using the Schrödinger wave function, one immediately gets $\mathbf{s}^{2,7}$

$$
f_{\mathrm{Sch}}(\infty)=1-\frac{1}{2}(Z \alpha)^{2}
$$

On the other hand, using $\psi_{\mathrm{FW}}^{0}$ as given in Eqs. (4) and (5), one would get ${ }^{17}$

$$
f_{\mathrm{FW}}(\infty)=1-\frac{1}{2} \frac{(Z \alpha)^{2}}{2 \gamma-1}
$$

Since $0<\gamma<1$, it is obvious that we will always have $f_{\mathrm{FW}}(\infty)<f_{\mathrm{Sch}}(\infty)$. In Fig. 1, we show a comparison between Eqs. (14) and (15), together with the exact relativistic results ${ }^{15}$ for different elements. It is not difficult to see that for heavy atoms, the SA can be very inaccurate and that the previous seemingly fine agreement ${ }^{2}$ with the exact calculation was only fortuitous. This implies that the higher-order terms in $\beta$ not included in the semirelativistic Hamiltonian should contribute significantly to such sum-rule calculations for the $K$ electrons of heavy elements. Thus we can conclude from the above examples that the SA, when consistently applied, may lead to results considerably smaller than the exact relativistic ones for large- $Z$ systems.

Recently, Smith ${ }^{7}$ has considered a similar comparison for the many-electron systems. Adopting an independent particle description, Smith has generalized the result derived in Ref. 2 to a system with $Z$ electrons to obtain

$$
f(\infty)=Z-\langle 0|T| 0\rangle / m c^{2},
$$

which turns out to be in agreement with previous results from direct calculations by Cromer and Liberman ${ }^{18}$ and Jensen. ${ }^{19}$ Smith then argued that usually the reduction of the forward atomic scattering factor from $Z$ is reported by applying the nonretarded $E 1$ approximation which leads to an extra factor $\frac{5}{3}$ in the correction term to $f(\infty)$ in (16), but with the inclusion of the $E 2$ oscillator strength and the retardation correction to the electricdipole transitions as in Eq. (13), the sum-rule calculations show good agreement with the measured anomalous scattering factor (see Fig. 1 of Ref. 7). However, in light of our present investigation for the one-electron system, we believe that Smith's agreement is again somewhat fortuitous, and a consistent evaluation of Eq. (16) using the Foldy-Wouthuysen-type wave function will lead to further deviation from the exact results. Moreover, the many-electron problem is further complicated for one is not clear regarding the relative importance of the contributions due to the correlation effects among the electrons and their effect on the $E 2$ oscillator strength and retardation correction to the $E 1$ transition. A further investigation of these effects would surely be interesting and would lead to a deeper understanding of the validity and limitation of the sum-rule approach.

The authors are grateful to Professor Joseph S. Levinger and Professor William McKinley for their comments on the earlier version of the manuscript and to Professor W. McKinley and Professor D. Y. Smith for the details of their calculations. This work was partially supported by the National Aeronautics and Space Administration under Grant No. NAG1577.
*Present address: Department of Physics and Department of Chemistry, State University of New York, Buffalo, New York 14260.

${ }^{1}$ A. Anholt and H. Gould, in Advances in Atomic and Molecular Physics, edited by D. Bates and B. Bedersen (Academic, New York, 1986), Vol. 22, p. 315.

${ }^{2}$ J. S. Levinger, M. L. Rustgi, and K. Okamoto, Phys. Rev. 106, 1191 (1957).

3 J. L. Friar and S. Fallieros, Phys. Rev. C 11, 274 (1975).

${ }^{4}$ K. M. Schmitt and H. Arenhovel, Z. Phys. A 320, 311 (1985).

${ }^{5}$ P. T. Leung, M. L. Rustgi, and S. A. T. Long, Phys. Rev. A 33,
2827 (1986).

${ }^{6} \mathrm{H}$. A. Bethe and E. E. Salpeter, Quantum Mechanics of Oneand Two-Electron Atoms (Springer, Berlin, 1957).

${ }^{7}$ D. Y. Smith, Phys. Rev. A 35, 3381 (1987).

${ }^{8}$ W. B. Payne and J. S. Levinger, Phys. Rev. 101, 1020 (1956).

${ }^{9}$ J. S. Levinger and M. L. Rustgi, Phys. Rev. 103, 439 (1956).

${ }^{10}$ J. D. Bjorken and S. D. Drell, Relativistic Quantum Mechanics (McGraw-Hill, New York, 1964).

${ }^{11}$ V. B. Berestetskii, E. M. Lifshitz, and L. P. Pitaevskii, Quantum Electrodynamics (Pergamon, New York, 1982).

${ }^{12}$ V. B. Berestetskii and L. D. Landau, Zh. Eksp. Teor. Fiz. 19, 
673 (1949).

${ }^{13}$ L. L. Foldy and S. A. Wouthysen, Phys. Rev. 78, 29 (1950).

${ }^{14}$ J. S. Levinger, Nuclear Photo-Disintegration (Oxford University Press, New York, 1960).

${ }^{15}$ V. Florescu and M. Gavrila, Phys. Rev. A 14, 211 (1976).

${ }^{16}$ G. Breit, Rev. Mod. Phys. 4, 504 (1932); 5, 91 (1933); M. GellMann, M. L. Goldberger, and W. Thirring, Phys. Rev. 95,
1612 (1954).

${ }^{17}$ Note that in this case the $\nabla^{2}$ term in Eq. (4) can be ignored since this would lead to a correction of order $\beta^{4}$ in $f(\infty)$. See Ref. 5 for more details.

${ }^{18}$ D. T. Cromer and D. Liberman, J. Chem. Phys. 53, 1891 (1970).

${ }^{19}$ M. S. Jensen, Phys. Lett. 74A, 41 (1979). 